\title{
Sobre a aplicação do conceito de bem-estar a peixes teleósteos e implicações para a piscicultura
}

\author{
Rui F. Oliveira \& Leonor Galhardo
}

Unidade de Investigação em Eco-Etologia, Instituto Superior de Psicologia Aplicada. Rua Jardim do Tabaco 34, 1149-041. Lisboa, Portugal.Rui.Oliveira@ispa.p)

RESUMO - A produção de peixes teleósteos em piscicultura tem vindo a aumentar nas últimas décadas. Embora exista legislação reguladora do bem-estar animal em contextos de produção animal que tende a abranger todos os vertebrados, e por consequência também as espécies piscícolas, o conhecimento acerca do bem-estar dos peixes é ainda reduzido. Noutras classes de vertebrados (i.e. mamíferos e aves), tradicionalmente utilizados em produção animal, o estudo do bem-estar animal permitiu no passado recente um conhecimento aprofundado do estado em que os animais se encontram e dos recursos mínimos de que necessitam nas explorações pecuárias, o que veio modelar normas de boas práticas, linhas de orientação e legislação acerca de como devem ser tratados em cativeiro. Estes estudos identificaram três dimensões centrais no conceito de bem-estar animal: (a) o funcionamento orgânico e a saúde dos animais; (b) o funcionamento mental e o sofrimento dos animais; e (c) a natureza dos animais e a capacidade destes poderem expressar em cativeiro o repertório comportamental típico da espécie. Relativamente aos peixes, os estudos disponíveis incidem sobretudo sobre aspectos de saúde orgânica e de respostas de estresse, pelo que dos três pilares do conceito de bem-estar animal apenas o primeiro tem sido considerado, faltando ainda integrar as três dimensões do bem-estar em estudos futuros. A presente comunicação tem como objectivo evidenciar como o bemestar animal é um conceito legitimamente extensível aos peixes, com base em dados recentes sobre a nocicepção, cognição e estresse social em peixes, que sugerem que estes são animais sencientes, e de como o conceito de bemestar deve ser tido em conta em actividades tão expressivas como a piscicultura.

Palavras Chave: aquacultura, bem-estar, cognição, estresse, nocicepção, peixes

\section{On the applicability of the concept of welfare to teleost fish and its implications for fish farming}

\footnotetext{
ABSTRACT - Fish farming has been increasing in the last decades. Although the current legislation on animal welfare of animals in production systems is applicable to all vertebrate species, including teleost fish, the knowledge on fish welfare is still very poor. In recent years, the study of animal welfare in other farmed vertebrates (i.e. mammals and birds) allowed a solid knowledge of the state of the animals and the minimal resources they need in production systems, which led to the implementation of good practice codes, guidelines and specific legislation on their maintenance in captivity. These studies have identified three core dimensions to the concept of animal welfare: (a) the health and biological functioning of animals; (b) suffering and the mental functioning of animals; (c) the nature of animals and the possibility for them to express in captivity the species-specific behavioural repertoire. Regarding fish the available studies mainly address biological health issues and the stress response, hence from the three pillars of the concept of animal welfare only the first one has been extensively studied, and an integration of the three dimensions is still lacking. The goal of this talk is to demonstrate that the concept of animal welfare is applicable to teleost fish, since recent data on nociception, cognition and social stress in fish suggest that they are sentient animals, and that fish welfare should be considered in human activities related to fish such as fish farming.
}

Key Words: aquaculture, cognition, fish, nociception, stress, welfare 


\section{$O$ conceito de bem-estar animal}

Ao interagir com espécies animais em diferentes contextos que vão da produção animal, à gestão de populações no âmbito de programas de conservação, passando pela utilização de espécies animais em experimentação animal e em parques zoológicos, o homem tem a responsabilidade de adaptar guias de boas práticas que garantam o bemestar dos animais em causa. Assim, o bem-estar animal é um conceito que surge no âmbito da interacção do homem com outros animais, e que emerge da atribuição de um estatuto moral aos animais. Deste modo, o conceito de bem-estar não tem cabimento se aplicado a animais selvagens no seu habitat, mas apenas quando factores antropogénicos influenciam a qualidade de vida dos animais, quer em cativeiro quer em meio natural.

A área científica do bem-estar animal tem como objectivo a caracterização da qualidade de vida dos animais e o desenvolvimento de estratégias que permitam o seu incremento quando os animais se encontram sob a responsabilidade de humanos. $\mathrm{Na}$ avaliação e na promoção da qualidade de vida dos animais têm sido tidos em conta três aspectos distintos: o seu funcionamento orgânico, as suas experiências mentais, e a expressão do seu comportamento natural (Fraser et al., 1997). Embora possa der dado um peso diferente a cada uma delas, estas três vertentes do bem-estar animal devem ser vistas de modo integrado e interdependente (Figura 1). Na realidade, no contexto da saúde humana esta é já a doutrina actual como pode ser facilmente ilustrado pela definição de sáude da Organização Mundial da Saúde da Organização das Nações Unidas como sendo um estado de completo bem-estar físico, mental e social, e não apenas a ausência de doença. No contexto dos animais o que o conceito de bem-estar tem de valor acrescentado em relação à saúde é precisamente a incorporação da dimensão mental dos animais. A capacidade consciente de sentir e as características cognitivas que lhe estão associadas constituem elementos-chave desta dimensão mental do bemestar animal (Broom, 1998; Appleby, 1999). Estados mentais negativos ou sofrimento (dor, medo, tédio, etc.) induzem mal-estar, enquanto que estados mentais positivos (alegria, conforto, prazer) propiciam o bem-estar no seu sentido positivo. Apesar das dificuldades de que se reveste o estudo dos estados mentais dos animais, existe actualmente um conjunto de evidências indirectas relacionadas com a anatomia, fisiologia, etologia e cognição que permitem a compreensão destes fenómenos em várias espécies de vertebrados (Broom, 1998; Appleby, 1999; Mendl \& Paul, 2004). As espécies para as quais existem evidências fortes que sugerem a capacidade dos indivíduos terem consciência de sensações e sentimentos (senciência) são aquelas às quais é atribuído estatuto moral. Deste modo, torna-se fulcral averiguar da existência desta característica em teleósteos para avaliar até que ponto é pertinente a aplicação a estes do conceito de bem-estar.

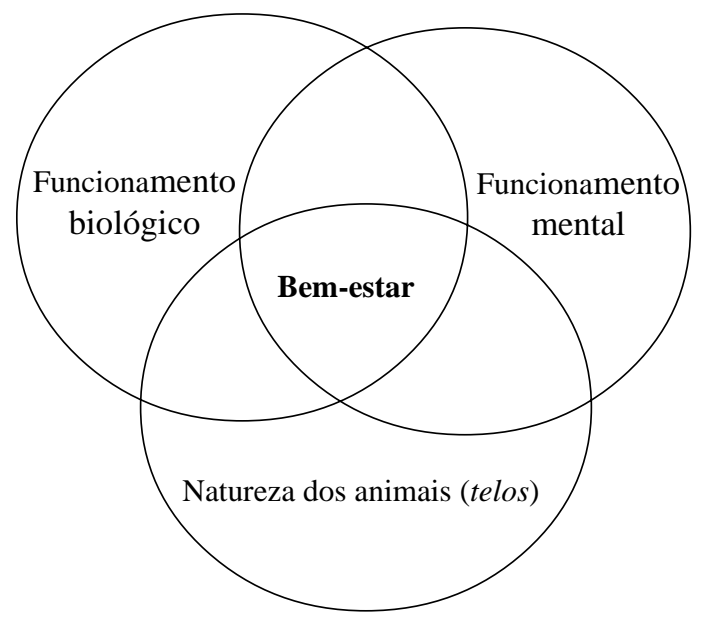

Figura 1 - O conceito de bem-estar animal resulta da integração de três dimensões: o funcionamento biológico dos animais, o seu funcionamento mental e a sua natureza (telos), a qual pode avaliada pela expressão do seu comportamento natural.

\section{Os teleósteos como animais sencientes}

$\mathrm{Na}$ avaliação da senciência de uma espécie existem pelo menos três factores que devem ser tidos em conta: a existência de um sistema nociceptivo que permita aos sujeitos uma percepção consciente (i.e. uma representação central) da dor, uma elevada complexidade cognitiva que sugira a capacidade de produção de representações elaboradas, e a ocorrência de estresse psicogénico que sugira que uma activação cognitiva da resposta a agentes de estresse.

\section{Nocicepção}

A resposta à dor em humanos tem dois componentes básicos: a resposta fisiológica, que pode 
ser um reflexo automático activado a nível periférico (medula espinal), e a experiência emocional da dor, que é processada a nível central no cérebro. Por analogia com os mecanismos de percepção da dor na nossa espécie, Smith \& Boyd (1991) enumeraram um conjunto de critérios que deveriam ser cumpridos para se estabelecer que um determinado animal tem a capacidade de percepcionar a dor que pressupõe sofrimento (Tabela 1).

Obviamente, quanto mais afastada, do ponto de vista filogenético, for a espécie menor será a probabilidade desta partilhar os mesmos componentes (homólogos) do sistema de percepção da dor com os humanos, pelo que não é de estranhar que entre os vertebrados apenas os mamíferos cumprem todos os critérios enunciados (Bateson, 1991; Smith \& Boyd, 1991). No entanto, nos últimos anos Lynn Sneddon e colaboradores publicaram um conjunto de estudos em salmonídeos que demonstram a ocorrência de nociceptores, a ligação destes a núcleos neurais no telencéfalo e a modulação da resposta a estímulos nocivos por opiáceos (Sneddon, 2003; Sneddon et al., 2003), o que no seu conjunto aponta para a presença da maioria dos componentes do sistema de nocicepção nos teleósteos (Tabela 1).

\section{Capacidades cognitivas}

O mais simples de todos os processos cognitivos relevantes para a senciência é a capacidade de sentir os estímulos internos e de percepcionar os externos (Duncan \& Petherick 1991). Esta capacidade está dependente da formação de representações mentais declarativas que, em humanos, envolvem a consciência. As representações declarativas implicam a atenção selectiva a estímulos, a capacidade de antecipar e possuir expectativas e o direccionamento flexível e integrado das respostas comportamentais (Chandroo et al., 2004). Em contraste, as representações processuais (ou implícitas) não são conscientes e traduzem respostas reflexivas, estando presentes em formas simples de condicionamento e habituação (Hampton \& Schwartz, 2004).

A investigação da memória envolvendo representações declarativas tem sido levada a cabo em animais de várias espécies, fornecendo importantes dados a respeito da ocorrência de fenómenos mentais conscientes. Um dos exemplos destes estudos é a investigação de memória episódica (evocação consciente de experiências passadas específicas) em gaios (Aphelocoma coerulescens). Clayton et al. (2003) concluíram que estas aves, armazenadoras de alimentos no meio natural, formam memórias integradas, flexíveis e dependentes de um único evento sobre onde, quando e que tipo de alimento escondem. Além disso aperceberam-se igualmente que animais com experiência de roubar comida armazenada, são capazes de atribuir esta característica a outros, ajustando o seu comportamento de armazenamento em função dessa antecipação. Outros estudos recentes incluem a averiguação de metacognição (o próprio saber o que sabe) em primatas, com resultados que evidenciam o uso de processos mentais conscientes (Mendl \& Paul, 2004).

Apesar de não ser abundante, a investigação efectuada em diversas espécies de peixes revelou comportamentos indiciadores de representações mentais declarativas e de uma memória e capacidade de aprendizagem complexas e flexíveis (Braithwaite \& Huntingford, 2004). Os mecanismos neuronais de algumas destas características cognitivas sugerem um certo grau de especialização e similaridade funcionais com os vertebrados terrestres, uma vez que a destruição de

Tabela 1 - Critérios propostos por Smith \& Boyd (1991) para avaliar a experiência da dor em animais, actualizados para os peixes teleósteos após os trabalhos de Sneddon e colaboradores.

\begin{tabular}{lccc}
\hline Critérios & \multicolumn{3}{c}{ Grupo taxonómico } \\
\cline { 2 - 4 } & Mamíferos & Aves & Teleósteos \\
\hline Presença de nociceptores & + & + & $? /+^{1}$ \\
Estruturas análogas do córtex humano & + & + & + \\
Ligação neural dos nociceptores ao SNC & + & $?$ & $? /+^{1}$ \\
Receptores opiáceos no SNC & + & + & + \\
Resposta a estímulos nocivos modificada por analgésicos & + & + & $? /+^{2}$ \\
Persistência de respostas a estímulos nocivos & + & + & $?$ \\
Condicionamento da resposta a estímulos nocivos & + & & + \\
\hline
\end{tabular}

1 = Sneddon et al., 2003; 2 = Sneddon, 2003. 
núcleos específicos do telencéfalo em peixes resulta em perturbações da orientação espacial, em particular da formação de mapas cognitivos, e de certas formas de aprendizagem, de forma similar à ocorrida noutros vertebrados em consequência de lesões do hipocampo (Salas et al., 1996).

\section{Estresse psicogénico}

A resposta a estímulos aversivos é um processo integrado que conta não só com a percepção do evento presente, mas também com a memória de experiências anteriores, o que confere à resposta ao estresse uma dimensão inevitavelmente psicológica (Barton 1997). Este aspecto foi demonstrado em tilápias do Nilo (Oreochromis niloticus), com um paradigma de condicionamento clássico no qual os animais foram treinados para associarem uma luz (estímulo condicionado) à ocorrência de um agente de estresse (confinamento). Após o período de aprendizagem, os peixes produzem o mesmo tipo de resposta hormonal (aumento dos níveis de cortisol) exclusivamente em resposta à exposição à luz (Moreira \& Volpato, 2004). Moreira et al. (2004) também demonstraram a existência de diferenças comportamentais e cognitivas (memória e aprendizagem) entre duas linhagens de trutas arco-íris seleccionadas pelas suas respostas baixas ou elevadas de cortisol a um agente de estresse standartizado. Também Schreck et al. (1995) já tinham mostrado, associando a remoção da água de salmão-real (Oncorhynchus tshawytscha) à distribuição de alimento, como o condicionamento positivo, contribuiu para atenuar a resposta fisiológica a estressores subsequentes (transporte). No seu conjunto estes três estudos sugerem uma componente cognitiva na activação da resposta de strese em peixes.

$\mathrm{O}$ contexto social em que os indivíduos vivem pode ser uma fonte de estresse originado pela dinâmica do estabelecimento de hierarquias, territorialidade, acasalamento, entre outros. Neste contexto, o estresse psicogénico pode ter três componentes: estados emocionais negativos (como o medo), processos de percepção que implicam o reconhecimento de conspecíficos e a capacidade de antecipação da presença ou de acções agonísticas por parte de conspecíficos (Chandroo et al., 2004). O estado de estresse crónico em que se encontram animais subor- dinados de várias espécies é com frequência resultado da percepção da presença de indivíduos dominantes. Este estado crónico, que provavelmente envolve medo, altera ou mesmo inibe a motivação desses animais para expressarem outros comportamentos (Chandroo et al., 2004).

Em conclusão, os dados disponíveis consubstanciam a existência de senciência em peixes, pelo que o estudo do seu bem-estar é da maior pertinência no contexto das actividades humanas com potenciais implicações para o seu bem-estar.

\section{Atividades humanas e bem-estar de peixes}

Uma vez reconhecida como válida a questão do bem-estar de peixes em função da sua senciência, existem duas posições filosóficas sobre a utilização de peixes em actividades humanas: a) a proposta dos direitos dos animais de Tom Regan segundo a qual nas espécies em que seja reconhecido um valor intrínseco aos seus indivíduos (i.e. sujeitos-de-uma-vida), estes têm direitos que não podem ser violados por interesses humanos, pelo que qualquer actividade da qual resultem prejuízos para os peixes deve ser descontinuada (Regan, 1984); b) a abordagem utilitarista de Peter Singer (1991), segundo a qual as consequências dos actos determinam a sua legitimidade, devendo existir igualdade de consideração de interesses iguais dos seres sencientes, independentemente da espécie. Como referido por Peter Sandøe e colaboradores (1997), são possíveis outras abordagens éticas baseadas na senciência, as quais combinam elementos do utilitarismo de Singer e dos direitos dos animais de Regan. A posição da maioria dos órgãos reguladores, vertida em códigos de boas práticas e na legislação de diferentes países reflecte um meio termo entre estas duas posições, em que se utiliza uma abordagem utilitarista até um certo limite a partir do qual se adopta uma lógica deontológica, segundo a qual o valor intrínseco dos animais prevalece sobre os interesses humanos. Esta prática tem implícito um modelo economicista de tomada de decisão que está explicitado na Figura 2. Este modelo baseia-se numa função utilitarista que define uma área de procedimentos aceitáveis e outra de acções inaceitáveis para com os animais em função da relação benefícios para os humanos (ganhos) vs. sofrimento animal (custos) e na existência de um

๑ 2007 Sociedade Brasileira de Zootecnia 
valor de corte que corresponde a um limiar de sofrimento animal acima do qual os custos se tornam inaceitáveis e provocam uma inflexão da função de tomada de decisão, que deixa de variar em função dos ganhos. Este modelo teórico levanta uma série de questões práticas uma vez que as variáveis em jogo não estão na mesma moeda e são difíceis de quantificar. Permite contudo formalizar um conjunto de regras que devem ser tidas em conta quando da implementação de normas de bem-estar em diferentes atividades.

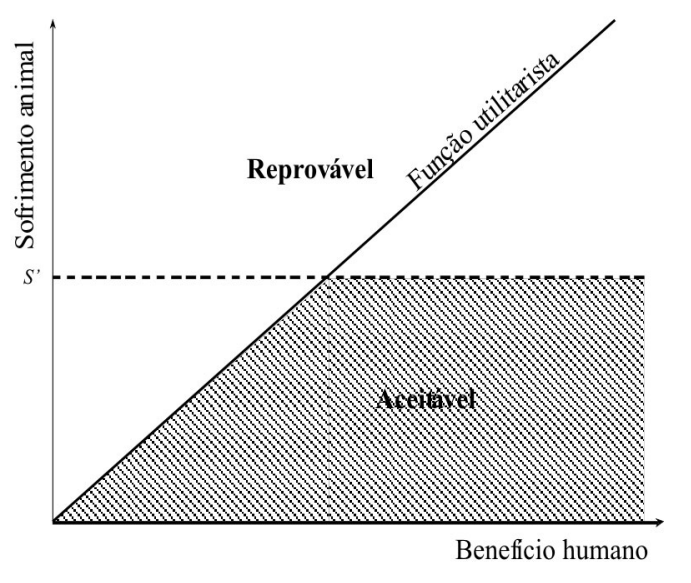

Figura 2 - Modelo de tomada de decisão para avaliar se uma determinada actividade humana é ou não aceitável de acordo com critérios de bem-estar animal. O modelo baseia-se numa função utilitarista, acima da qual (i.e. custos para animais > benefícios para humanos) as actividades não devem ser permitidas e abaixo da qual são aceitáveis (i.e. benefícios para humanos > custos para animais), e num valor de corte $\left(S^{\prime}\right)$ deontológico que define um limiar de sofrimento animal acima do qual as actividades não devem ser permitidas independentemente do valor do benefício humano.

Os peixes são utilizados em diversas atividades humanas cuja sustentabilidade do ponto de vista do bem-estar animal depende de uma análise de custos-benefícios. As principais atividades humanas com implicações para o bem-estar dos peixes incluem a sua captura em pesca comercial e desportiva, produção intensiva em aquacultura, utilização como animais de companhia, manutenção em aquários públicos para educação ambiental e recreação, utilização em investigação científica e a degradação ambiental antropogénica. Não pretendendo com esta comunicação fazer juízos de valor sobre as atividades que são aceitá- veis ou inaceitáveis, mas antes ilustrar como se podem tentar avaliar efeitos negativos no bemestar dos peixes - que devem ser tidos em consideração e contrastados com os benefícios das atividades visadas segundo o modelo acima exposto listámos na Tabela 2 potenciais custos e ganhos das principais actividades humanas com potencial impacto no bem-estar dos peixes (FSBI, 2002).

\section{Avaliação do bem-estar em peixes}

Para quantificar os potenciais impactos negativos das actividades humanas no bem-estar dos peixes torna-se necessário desenvolver metodologias de avaliação do bem-estar. Estas avaliam o estado dos animais a nível físico, da sua fisiologia e do seu comportamento. Idealmente, estes três níveis devem ser considerados em conjunto na avaliação do bem-estar.

\section{Saúde e bem-estar}

O funcionamento orgânico e a saúde são um dos aspectos fundamentais do bem-estar animal. Doenças, ferimentos, malformações e má nutrição são as principais ameaças ao equilíbrio orgânico dos animais. Em geral, os sinais positivos de saúde provêm de um bom aspecto físico, alimentação regular, taxas de crescimento e reprodução normais, boa longevidade e taxas de mortalidade reduzidas (Duncan \& Fraser 1997). No entanto, as causas da doença são complexas e multifactoriais na maior parte dos casos, pelo que a relação entre doença e bem-estar não é necessariamente biunívoca. Assim, não se pode generalizar a ideia de que problemas de saúde são invariavelmente indicadores de más condições de bem-estar em que os animais se encontram.

\section{Estresse e bem-estar}

$\mathrm{O}$ estresse pode ser considerado como um conjunto de respostas não específicas do organismo a situações que ameaçam desequilibrar a sua homeostase (Barton, 2002; FSBI, 2002). Os agentes de estresse em peixes podem ser de vários tipos, nomeadamente de natureza física, como o transporte, o confinamento ou manuseamento; de natureza química, como os contaminantes, o baixo teor de oxigénio ou o $\mathrm{pH}$ reduzido; e os percepcionados pelos animais, como a presença de predadores ou de conspecíficos não-familiares (Barton 
Tabela 2 - Principais actividades humanas com potencial impacto no bem-estar de peixes (adaptado de FSBI, 2002).

\begin{tabular}{|c|c|c|}
\hline Actividade & Potenciais benefícios humanos & $\begin{array}{l}\text { Potenciais custos em bem-estar } \\
\text { animal }\end{array}$ \\
\hline Pesca comercial e desportiva & $\begin{array}{l}\text { - Alimentação } \\
\text { - Economia: sectores das Pescas e Turismo. }\end{array}$ & $\begin{array}{l}\text { - Dor e estresse associados às técnicas de } \\
\text { captura e de abate. } \\
\text { - Destruição de tecidos quando é utilizado } \\
\text { isco em anzóis. }\end{array}$ \\
\hline Produção intensiva & $\begin{array}{l}\text { - Alimentação } \\
\text { - Economia: Aquacultura }\end{array}$ & $\begin{array}{l}\text { - Fraca qualidade da água. } \\
\text { - Elevadas densidades (maior exposição } \\
\text { a agentes patogénicos e ambiente social } \\
\text { inapropriado). } \\
\text { - Privação alimentar (em períodos de } \\
\text { colheita e de quarentena) } \\
\text { - Dor e estresse associado a técnicas de } \\
\text { maneio, transporte e abate. }\end{array}$ \\
\hline $\begin{array}{l}\text { Peixes ornamentais como animais } \\
\text { de companhia }\end{array}$ & $\begin{array}{l}\text { - Bem-estar humano } \\
\text { - Educação zoófila que melhora atitudes para } \\
\text { com animais. } \\
\text { - Economia: Pesca e Aquacultura de espécies } \\
\text { ornamentais. }\end{array}$ & $\begin{array}{l}\text { - Utilização de venenos em doses sub- } \\
\text { letais na captura. } \\
\text { - Efeitos nocivos de selecção artificial. } \\
\text { - Risco de introdução de espécies } \\
\text { exóticas. } \\
\text { - Fraca qualidade da água e más con- } \\
\text { dições de manutenção (incluindo dieta } \\
\text { inapropriada) de aquários amadores. } \\
\text { - Ambientes sociais desajustados com } \\
\text { privação social (e.g. peixe vermelho } \\
\text { mantido em aquários individuais). }\end{array}$ \\
\hline Exibição em aquários públicos & $\begin{array}{l}\text { - Recreio. } \\
\text { - Educação ambiental. } \\
\text { - Conservação da Natureza. } \\
\text { - Economia: Turismo e Lazer. }\end{array}$ & $\begin{array}{l}\text { - Exibição de espécies bandeira que por } \\
\text { vezes têm necessidades comporta- } \\
\text { mentais incompatíveis com condições } \\
\text { de cativeiro (e.g. tubarão-baleia). } \\
\text { - Ambientes sociais desajustados com } \\
\text { coexistência de espécies de diferentes } \\
\text { habitats nos mesmos aquários e/ou } \\
\text { coexistência de presas e predadores nos } \\
\text { mesmos aquários. } \\
\text { - Perturbações por visitantes (e.g. } \\
\text { fotografias com flash). }\end{array}$ \\
\hline Investigação científica & $\begin{array}{l}\text { - Progresso do conhecimento científico. } \\
\text { - Saúde humana. } \\
\text { - Saúde animal. } \\
\text { - Economia: Inovação e Desenvolvimento. }\end{array}$ & $\begin{array}{l}\text { - Exposição experimental a estímulos / } \\
\text { tratamentos nocivos (e.g. exposição a } \\
\text { predadores; inoculação de agentes } \\
\text { patogénicos, etc.) } \\
\text { - } \text { Dor e estresse associados a procedi- } \\
\text { mentos experimentais (e.g. cirurgia) e } \\
\text { maneio em laboratório (e.g. técnicas de } \\
\text { marcação individual). } \\
\text { - Pesca eléctrica. }\end{array}$ \\
\hline $\begin{array}{l}\text { Degradação ambiental } \\
\text { antropogénica }\end{array}$ & $\begin{array}{l}\text { - Produção de energia hidroeléctrica em } \\
\text { barragens. } \\
\text { - Utilização de barragens para recreio e lazer; } \\
\text { irrigação de campos de cultivo. } \\
\text { - Actividades turísticas associadas a } \\
\text { barragens. } \\
\text { - Utilização de transporte marítimo e de } \\
\text { embarcações motorizadas em diversas } \\
\text { actividades. } \\
\text { - Efluentes industriais com contaminantes } \\
\text { (e.g. disruptores endócrinos). } \\
\text { - Economia: Energia, Transportes, Recreio e } \\
\text { Lazer, Turismo, Atividade Industrial. }\end{array}$ & $\begin{array}{l}\text { - Barragens criam barreiras artificiais a } \\
\text { espécies migratórias. } \\
\text { - Poluição sonora por tráfego marinho de } \\
\text { embarcações motorizadas. } \\
\text { - Fragmentação ambiental. } \\
\text { - Perturbações por visitantes (e.g. eco- } \\
\text { turismo). }\end{array}$ \\
\hline
\end{tabular}


1997). A exposição aos agentes de estresse pode ser de curta ou de longa duração, e pode ter diferentes intensidades. A exposição moderada a estes agentes pode produzir nos peixes uma resposta adaptativa, que restitui o equilíbrio ao organismo. Contudo, se estes estiverem sujeitos a agentes de estresse intensos ou prolongados, a resposta pode tornar-se maladaptativa, com consequências negativas para o seu estado de saúde. Conte (2004) reconhece que o estresse é um dos principais factores responsáveis pela ocorrência de doenças e mortalidade em aquacultura. A intensidade e duração da resposta a estímulos adversos depende das espécies, estirpes, ou stocks considerados, bem como do facto de se tratar de animais nascidos em meio natural ou em cativeiro. Também as condições ambientais, de desenvolvimento ou genéticas podem gerar uma maior ou menor susceptibilidade a agentes de estresse, influenciando o tipo de respostas geradas (Barton, 2002). Barton (1997) chama a atenção para a necessidade de ter estes aspectos em consideração quando se interpretam os resultados de análises ao cortisol. O mesmo autor (Barton, 2002) refere a relevância da investigação de taxas metabólicas, imunocompetência e reprodução na compreensão dos contextos em que o estresse ocorre.

Finalmente, deve-se referir que a utilização avulsa de níveis de cortisol como indicador de bem -estar deve ser evitada uma vez que valores baixos de cortisol podem não ser indicadores de bem-estar mas sim de exaustão da capacidade de secreção do tecido inter-renal; assim como elevados níveis de cortisol podem estar associados à preparação do organismo para actividade física (FSBI, 2002). Assim, deve ser reconhecido que o estresse fisiológico não é necessariamente indicador de sofrimento e que por si só medidas de cortisol ou outros indicadores de estresse são insuficientes para uma caracterização fidedigna do bem-estar.

\section{Comportamento e bem-estar}

O terceiro aspecto considerado na avaliação do bem-estar animal é a questão da possibilidade de os animais poderem expressar repertório comportamental típico da espécie. Contudo, tal como alguns autores põem em evidência (Fraser et al., 1997; Dawkins, 2004), certos comportamentos naturais podem já não ser relevantes para os animais quando mantidos em condições artificiais, ou podem mesmo ser indicadores de mal-estar (e.g. fuga a predadores). Por isso, mais do que a questão da natureza dos animais, o importante será a compreensão da forma como o comportamento está relacionado com a saúde e com o que o animal deseja ou não em cada momento (i.e. necessidades comportamentais; Dawkins 2004).

Uma vez que a recolha metódica de dados fisiológicos, bioquímicos e comportamentais implica um esforço de amostragem razoável, requer um grande investimento de tempo e uma logística pesada, não compatíveis com rotinas quotidianas em aquários públicos ou em estações de aquacultura, a Fisheries Society of the British Isles sugeriu a utilização dos seguintes indicadores práticos de bem-estar em peixes (FSBI, 2002):

1. Alterações do padrão de coloração - do corpo e dos olhos podem ser induzidas por estresse.

2. Mudanças na taxa de movimentos operculares - maiores necessidades de oxigénio associadas a estresse levam a uma maior necessidade de irrigação das brânquias. Pode ser combinado com avaliação visual do estado das brânquias.

3. Alteração da actividade de natação e de outros comportamentos - Atividade excessiva ou imobilidade, tentativas de fuga e comportamentos de esfregar o corpo para remover ectoparasitas são potenciais indicadores de problemas.

4. Anorexia-perda de apetite potencialmente relacionada com estresse.

5. Redução da taxa de crescimento - Pode ser indicador de estresse crónico.

6. Perda de factor de condição - indica uma redução da massa corporal.

7. Anomalias morfológicas - indicadoras potenciais de estresse durante o desenvolvimento larvar.

8. Cicatrizes - são usualmente indicadores de bem-estar negativo, como por exemplo o ataque por conspecíficos.

9. Doenças - Uma vez que as doenças de organismos aquáticos dependem em grande medida das condições ambientais, o aparecimento de doenças em peixes de cativeiro sugere problemas de manutenção.

10. Inibição reprodutora - potencial indicador de estresse crónico.

๑ 2007 Sociedade Brasileira de Zootecnia 
A utilização desta lista deve ser feita com cuidado uma vez que a sua aplicabilidade varia com a espécie e com o contexto no qual a avaliação de bem-estar é efectuada.

\section{Bem-estar de peixes em aquacultura}

Tendo em atenção a aquacultura é um dos sectores preferênciais de intervenção zootécnica e que é um dos sectores da produção animal que mais rapidamente se expandiu por todo o globo, sendo de importância económica fulcral em praticamente todos os continentes (Conte, 2004), serão de seguida discutidos alguns aspectos de bem-estar de peixes neste contexto.

A produção intensiva de peixes implica sistemas concebidos para produzir o máximo ao menor custo. Contudo, actualmente é cada vez mais reconhecido que a alta produtividade deve ser compatibilizada com boas práticas nos cuidados prestados aos animais. A sua saúde e bem-estar ocupam assim uma importância crescente nas preocupações relacionadas com as técnicas de produção adoptadas (Schwedler \& Johnson, 2000). Aspectos como o maneio, o alojamento e os procedimentos adoptados relativamente ao transporte e abate são as áreas que por ventura têm um maior impacto no bem-estar dos peixes em sistemas de produção.

\section{Maneio}

A manutenção do funcionamento biológico normal e a resistência a doenças requer uma dieta nutricionalmente equilibrada, e ajustável às necessidades específicas dos peixes (Fletcher, 1997). Contudo, o conhecimento acerca do que constitui uma dieta equilibrada é ainda muito restrito, sobretudo para os peixes recentemente usados em aquacultura (Schwedler \& Johnson, 2000). Embora os períodos de privação alimentar dos peixes possam não ter o mesmo impacto no seu equilíbrio, por serem animais ectotérmicos, a consideração pela sua motivação alimentarem é essencial na preservação do bem-estar. Com base em dados de taxas de crescimento e conversão, Alanärä (1996) refere o uso de alimentadores activados pelos animais como tendo potencial em aquacultura. As consequências de privação alimentar prolongada incluem a erosão da barbatana dorsal de truta arco-íris, o aumento da glucose plasmática no salmão, e a perda de peso e condição física em várias outras espécies (FSBI, 2002).

A captura para controlo de doenças ou transporte ou a separação dos animais por tamanhos (grading), bem como outros procedimentos que impliquem a manipulação física, são actividades causadoras de estresse, pelo que carecem de investigação continuada e aplicação de métodos que minimizem este impacto (FSBI, 2002). Técnicas de condicionamento operante podem ser uma possibilidade para reduzir ou mesmo eliminar o estresse presente em situações de maneio, tal como foi evidenciado por Schreck et al. (1995) que associaram um reforço positivo (alimento) à captura e transporte de salmão-real. Também Lines \& Frost (1999) descrevem uma série de técnicas de controlo e inspecção selectiva de animais baseadas nas próprias características sensoriais e comportamentais destes.

O controlo de doenças em aquacultura passa pelo controlo da exposição a agentes patogénicos e de situações que possam causar ferimentos aos animais, mas passam sobretudo pelo controlo da exposição a agentes de estresse (Schwedler \& Johnson, 2000; Conte, 2004).

\section{Alojamento}

As concentrações de oxigénio, de dióxido de carbono e de nitrogénio dissolvidos na água, a salinidade e o pH, a taxa de circulação da água, a temperatura e os regimes de luminosidade são os factores ambientais mais críticos à manutenção do equilíbrio dos peixes. Assim, a qualidade da água e os factores ambientais associados é uma das áreas que tem recebido mais atenção quer por parte da investigação sobre o estresse em peixes, quer pela própria indústria (FSBI, 2002). Para obtenção de valores óptimos é necessário ter em conta as características naturais dos animais, mas também a interacção destes factores com outros, como o espaço disponível e o ambiente social (Conte, 2004; Schwedler \& Johnson, 2000). A ocorrência de poluentes químicos na água, frequentemente usados pela própria actividade de produção, também é um aspecto que carece de controlo na protecção dos peixes. Costello et al. (2001) revêem os principais químicos usados na indústria Européia e produzem linhas de orientação para melhorar a prática do seu uso.

A densidade populacional nos sistemas de criação de peixes é um dos factores mais críticos

\footnotetext{
๑) 2007 Sociedade Brasileira de Zootecnia
} 
em aquacultura e no bem-estar dos animais. Densidades inadequadas podem promover a agressividade, e o excesso populacional incrementa a competição e influencia negativamente a qualidade da água. A falta de espaço para nadar é também prejudicial a muitas espécies (Schwedler \& Johnson, 2000). A dimensão óptima dos grupos depende das características comportamentais dos animais (em particular, a tendência para formar cardumes, ou a territorialidade). Em geral, densidades demasiado elevadas têm um efeito prejudicial (e.g. salmão, FSBI 2002). Alguns dos indicadores de excesso populacional prolongado em trutas incluem a redução das taxas de conversão e crescimento, a redução da condição física, e a erosão das barbatanas dorsais (Ellis et al., 2002). No entanto, os mesmos autores referem nem sempre existir evidência de estresse em condições de densidade elevada nesta espécie. Com efeito, esta relação pode não ser linear, pois em determinados contextos a densidade baixa em trutas pode mesmo tornar-se prejudicial (FSBI, 2002). Para outras espécies, geralmente territoriais, as densidades altas parecem ajustar-se melhor a um ambiente com níveis de agressividade baixos. Este é o caso de espécies como o salvelino-ártico e o peixe-gato (FSBI, 2002). Para espécies, como a tilápia do Nilo (Oreochromis niloticus), existe um óptimo populacional, pois densidades baixas promovem agressividade entre machos, e densidades demasiado elevadas têm efeitos negativos a nível reprodutivo (Bhujel, 2000).

Devido às hierarquias sociais que se estabelecem em certas espécies, a composição dos grupos sociais deve ser igualmente objecto de atenção em aquacultura para reduzir a agressividade de animais dominantes e o estresse e, com frequência, subnutrição crónicos em subordinados (FSBI, 2002). A prevenção de estresse agudo e de ameaças à integridade física dos peixes passa também pelo controlo de predadores, como focas, aves, etc., em aquaculturas (FSBI, 2002).

$\mathrm{O}$ enriquecimento ambiental em peixes de aquacultura é um assunto praticamente não abordado. Contudo, face às características ecológicas de algumas espécies um certo grau de complexidade ambiental poderia ser importante (FSBI, 2002). Seria de inferir que locais de abrigo, marcos territoriais, uso de diferentes substratos, correntes de água, formas estratégicas de distri- buição de alimento (e.g. Alanärä 1996), entre outros poderiam ter um papel relevante nas preferências e opções dos animais, incrementando assim o seu bem-estar.

\section{Transporte e abate}

Os factores mais críticos a considerar em relação ao transporte são o manuseamento dos animais (captura) e o controlo dos factores ambientais da água durante o transporte, já que os animais são transportados em altas densidades (Conte, 2004). $\mathrm{O}$ estresse fisiológico provocado pelo manuseamento e transporte dos peixes permanece durante 6 horas a um dia (Schreck et al., 1997). Como mencionado atrás, técnicas de condicionamento podem ajudar os animais a reduzir a sua reacção negativa a estes procedimentos (Schreck et al., 1995).

As técnicas de abate de peixes têm sido alvo de inúmeros estudos, com vários objectivos, entre os quais os de promover o controlo de qualidade, a eficiência e a segurança dos procedimentos (Conte, 2004). Vários trabalhos têm também o objectivo de minimizar o tempo necessário para produzir a morte, e implicitamente reduzir o medo e a dor que os animais possam sentir (e.g. Lambooij et al., 2002). Alguns exemplos de métodos de abate incluem o atordoamento eléctrico seguido de decapitação, o golpe letal na cabeça, o atordoamento percussivo, o atordoamento pelo frio e a remoção da água. Os atordoamentos eléctricos e percussivos, aplicados somente na cabeça, parecem ser em geral os métodos causadores de menos perturbação, a julgar por dados comportamentais, por indicadores de reflexos cerebrais e pela qualidade da carcaça. A morte por asfixia e com recurso ao gelo parecem ser os métodos menos aceitáveis (Conte, 2004).

\section{Conclusão}

As evidências apresentadas acerca da percepção da dor nos peixes, da dimensão psicológica da resposta a agentes de estresse que apresentam, e da sua complexidade cognitiva, sugerem fortemente a existência de senciência nestes animais, o que legitima a aplicação do conceito de bemestar aos teleósteos. Ao mesmo tempo, estes aspectos do funcionamento físico, fisiológico e comportamental são de relevância fundamental na

\footnotetext{
๑ 2007 Sociedade Brasileira de Zootecnia
} 
avaliação do bem-estar, podendo contribuir para a produção de recomendações na manutenção e maneio de peixes em cativeiro, nomeadamente em aquacultura.

\section{Agradecimentos}

Os autores agradecem o amável convite da Sociedade Brasileira de Zootecnia para participar na 44 ${ }^{\mathrm{a}}$. Reunião Anual da SBZ, em particular ao Prof. Dr. Mateus Paranhos da Costa (UNESP/ Jaboticabal). A preparação deste trabalho foi financiada pelo Programa Plurianual da Fundação para a Ciência e a Tecnologia (FCT) (UI\&D 331/ 2001) no âmbito do Programa FEDER da EU. A Dra. Leonor Galhardo é bolseira de doutoramento da FCT (SFRH/BD/16162/2004).

\section{Literatura citada}

ALANÄRÄ, A. The use of self-feeders in rainbow trout (Oncorhynchus mykiss) production. Aquaculture, v.145, p.120, 1996.

APPLEBY, M. What should we do about animal welfare? Oxford: Blackwell Science, 1999.

BARTON, B.A. Stress in finfish: past, present and future - a historical perspective. In: Iwana, G.K.; Pickering, A.D.; Sumpter, J.P.; Schreck, C.B. (eds) Fish Stress and Health in Aquaculture. Society for Experimental Biology, Seminar Series 62, Cambridge: Cambridge University Press, 1997. p1-33.

BARTON, B.A. Stress in fishes: a diversity of responses with particular reference to changes in circulating corticosteroids. Integrative and Comparative Biology, v.42, p.517-525, 2002.

BATESON, P. Assessment of pain in animals. Animal Behaviour, v. 42, p.827-839, 1991.

BHUJEL, R.C. A review of strategies for the management of Nile tilapia (Oreochromis niloticus) broodfish in seed production systems, especially hapa-based systems. Aquaculture, v.181, p.37-59, 2000.

BRAITHWAITE, V.A.; HUNTINGFORD, F.A. Fish and welfare: do fish have the capacity for pain perception and suffering? Animal Welfare, v.13 suppl, p.S87-92, 2004.

BROOM, D.M. Welfare, Stress and the Evolution of Feelings, Advances in the Study of Behaviour, v.27, p.317-403, 1998. CHANDROO, K.P.; DUNCAN, I.J.H.; MOCCIA, R.D. Can fish suffer?: perspectives on sentience, pain, fear and stress. Applied Animal Behaviour Science. v.86, p.225-250, 2004.

CLAYTON, N.S.; BUSSEY, T.J.; DICKINSON, A. Can animals recall the past and plan for the future? Nature Reviews/ Neuroscience, v.4, p.685-691, 2003.

CONTE, F.S. Stress and the welfare of cultured fish. Applied Animal Behaviour Science, v.86, p.205-223, 2004.

COSTELLO, M.J.; GRANT, A.; DAVIES, I.M. et al. The control of chemicals used in aquaculture in Europe. Journal of Applied Ichthyology, v.17, n.4, p.173-180, 2001.

DAWKINS, M.S. Using behaviour to assess animal welfare. Animal Welfare, v.13 Suppl, p.S19-30, 2004.

DUNCAN, I.J.H.; FRASER, D. Understanding Animal Welfare. In: Appleby, M. \& Hughes, B.O. (eds) Animal Welfare. London: CABI Publishing, 1997. p.19-32.

DUNCAN, I.J.H.; PETHERICK, J.C. The implications of cognitive processes for animal welfare. Journal of Animal Science, v.69, p.5017-5022, 1991.

๑ 2007 Sociedade Brasileira de Zootecnia
ELLIS, T.; NORTH, B.; SCOTT, A.P. et al. The relationships between stocking density and welfare in farmed rainbow trout. Journal of Fish Biology, v.61, n.3, p.493-531, 2002.

FLETCHER, T.C. Dietary effects on stress and health. In: Iwana, G.K.; Pickering, A.D.; Sumpter, J.P.; Schreck, C.B. (eds) Fish Stress and Health in Aquaculture. Society for Experimental Biology, Seminar Series 62, Cambridge: Cambridge University Press, 1997. p.223-246.

FRASER, D.; WEARY, D.M.; PAJOR, E.A. et al. A scientific conception of animal welfare that reflects ethical concerns. Animal Welfare, v.6, p.187-206, 1997.

FSBI Fish Welfare (Briefing Paper 2). Fisheries Society of the British Isles. Cambridge: Granta Information Systems, 2002. http://www.le.ac.uk/biology/fsbi/briefing.html

HAMPTON, R.R.; SCHWARTZ, B.L. Episodic memory in nonhumans: what, and where, is when? Current Opinion in Neurobiology, v.14, p.192-197, 2004.

LAMBOOIJ, E.; VAN DE VIS, J.W.; KLOOSTERBOER, R.J. et al. Welfare aspects of live chilling and freezing of farmed eel (Anguilla anguilla L.): neurological and behavioural assessment. Aquaculture, v.210, p.159-169, 2002.

LINES, J.A.; FROST, A.R. Review of opportunities for low stress and selective control of fish. Aquacultural Engineering, v.20, p.211-230, 1999.

MENDL, M.; PAUL, E.S. Consciousness, emotion and animal welfare: insights from cognitive science. Animal Welfare, v.13, Suppl:S17-26, 2004.

MOREIRA, P.S.A.; VOLPATO, G.L. Conditioning of stresse in Nile tilapia. Journal of Fish Biology, v.64, p.961-969, 2004. MOREIRA, P.S.A.; PULMAN, K.G.T.; POTTINGER, T.G. Extinction of a conditioned response in rainbow trout selected for high or low responsiveness to stress. Hormones and Behaviour, v.46, p.450-457, 2004.

REGAN, T. The Case for Animal Rights. London: Routledge, 1984.

SALAS, C.; BROGLIO, C.; RODRIGUEZ, F. et al. Telencephalic ablation in goldfish impairs performance in a 'spatial constancy' problem but not in a cued one. Behavioural Brain Research, v.79, p.193-200, 1996.

SANDØE, P.; CRISP, R.; HOLTUG, N. Ethics. In: Appleby, M. $\&$ Hughes, B.O. (eds) Animal Welfare. London: CABI Publishing, 1997. p.3-18.

SCHRECK, C.B.; JOHNSSON, L.; FEIST, G. et al. Conditioning improves performance of juvenile Chinook salmon, Oncorhynchus tshawytscha, to transportation stress. Aquaculture, v.135, p.99-110, 1995.

SCHRECK, C.B.; OLLA, B.L.; DAVIS, M.W. Behavioral responses to stress. . In: Iwana, G.K., Pickering, A.D. Sumpter, J.P., Schreck, C.B. (eds) Fish Stress and Health in Aquaculture. Society for Experimental Biology, Seminar Series 62, Cambridge: Cambridge University Press, 1997. p.145-170.

SCHWEDLER, T.E.; JOHNSON, S.K. Responsible care and health maintenance of fish in commercial aquaculture. Animal Welfare Information Center Bulletin, Winter 1999/ 2000, v.10, p.3-4.

SINGER, P. Animal Liberation. 2nd edn. London: Thorsons, 1991.

SMITH, J.A. \& BOYD, K.M. Lives in the Balance: the Ethics of using Animals in Biomedical Research. Oxford: Oxford University Press, 1991.

SNEDDON, L.U. The evidence for pain in fish: the use of morphine as an analgesic. Applied Animal Behaviour Science. v.83, p153-162, 2003.

SNEDDON, L.U.; BRAITHWAITE, V.A.; GENTLE, M.J. Do fish have nociceptors? Evidence for the evolution of a vertebrate sensory system. Proc. R. Soc. London, v.B270, p.1115-1121, 2003. 